\title{
An integrative analysis of cellular contexts, miRNAs and mRNAs reveals network clusters associated with antiestrogen-resistant breast cancer cells
}

Seungyoon Nam ${ }^{1,2^{*}}$, Xinghua Long ${ }^{2,3}$, ChangHyuk Kwon ${ }^{1}$, Sun Kim ${ }^{4}$ and Kenneth P Nephew ${ }^{2}$

\begin{abstract}
Background: A major goal of the field of systems biology is to translate genome-wide profiling data (e.g., mRNAs, miRNAs) into interpretable functional networks. However, employing a systems biology approach to better understand the complexities underlying drug resistance phenotypes in cancer continues to represent a significant challenge to the field. Previously, we derived two drug-resistant breast cancer sublines (tamoxifen- and fulvestrant-resistant cell lines) from the MCF7 breast cancer cell line and performed genome-wide mRNA and microRNA profiling to identify differential molecular pathways underlying acquired resistance to these important antiestrogens. In the current study, to further define molecular characteristics of acquired antiestrogen resistance we constructed an "integrative network". We combined joint miRNA-mRNA expression profiles, cancer contexts, miRNA-target mRNA relationships, and miRNA upstream regulators. In particular, to reduce the probability of false positive connections in the network, experimentally validated, rather than prediction-oriented, databases were utilized to obtain connectivity. Also, to improve biological interpretation, cancer contexts were incorporated into the network connectivity.

Results: Based on the integrative network, we extracted "substructures" (network clusters) representing the drug resistant states (tamoxifen- or fulvestrant-resistance cells) compared to drug sensitive state (parental MCF7 cells). We identified un-described network clusters that contribute to antiestrogen resistance consisting of miR-146a, -27a, $-145,-21,-155,-15 a,-125 b$, and let-7s, in addition to the previously described miR-221/222.
\end{abstract}

Conclusions: By integrating miRNA-related network, gene/miRNA expression and text-mining, the current study provides a computational-based systems biology approach for further investigating the molecular mechanism underlying antiestrogen resistance in breast cancer cells. In addition, new miRNA clusters that contribute to antiestrogen resistance were identified, and they warrant further investigation.

Keywords: Bioinformatics, miRNA, Network, Breast cancer, Antiestrogen resistance

\footnotetext{
* Correspondence: seungyoon.nam@ncc.re.kr

${ }^{1}$ Cancer Genomics Branch, National Cancer Center, Goyang-si, Gyeonggi-do 410-769, Korea

2Department of Cellular and Integrative Physiology, Medical Sciences

Program, Indiana University School of Medicine, Bloomington IN 47405, USA

Full list of author information is available at the end of the article
} 


\section{Background}

Endocrine therapy is a highly effective form of adjuvant therapy for hormone sensitive breast cancer. Currently, the three classes of commonly used drugs for adjuvant endocrine therapy are selective estrogen receptor modulators (SERMs, e.g., tamoxifein), selective estrogen receptor down-regulators (SERDs, e.g., fulvestrant), and aromatase inhibitors (AIs). Unfortunately, tumor cells often develop resistance to endocrine therapy [1], representing a major obstacle limiting the success of breast cancer treatment. To better understand the biology and molecular mechanisms that underlie endocrine resistance, we and others have developed tamoxifen- and fulvestrant-resistant breast cancer cell models $[1,2]$. We demonstrated that dramatically different molecular mechanisms underlie progression to resistance to tamoxifen (henceforth, MCF7-T) and fulvestrant (henceforth, MCF7-F) and also identified specific genes and biochemical pathways associated with SERM- and SERD-resistance.

Recently, microRNAs (miRNAs), a novel noncoding RNA class [3-5], have been shown to be key regulators of various biological processes and diseases $[3,6]$. In breast cancer, alterations in expression of miRNAs appear to play important roles in drug resistance $[7,8]$ and thus may represent new therapeutic targets. Furthermore, the role of specific miRNAs in antiestrogen (fulvestrant, tamoxifen) resistant breast cancer has been investigated by us [9] and others [10,11], and both Rao et al. [9] and Miller et al. [10] demonstrated a critical role for miR-221/222 in SERM and SERD resistances as well as a key role in estrogen receptor alpha (ER $\alpha)$ biology and function. In this followup study, we took a global approach to further investigate the role of miRNAs in resistance to these important endocrine therapies.

Although methods for the functional analysis of miRNAs are publicly available [12-14], systematic global view $[15,16]$ of the networks of these key epigenetic regulators has not been fully explored. Systems biology [17] approaches have recently been used to examine miRNA-mediated pathogenic dys-regulation $[15,18,19]$ and oestrogen-regulated miRNAs [20]; however, this approach has only recently been used to investigate breast cancer drug resistance [21], one of the most lethal cancers in women. Here, we present an integrative view of "antiestrogen resistance-related miRNA-mRNA regulation" and discuss functional roles of this previously un-described network. The network was reconstructed by combining cancer contexts and expression profiles for miRNAs and mRNAs. Furthermore, in order to minimize false positives in the network construction [4], we utilized experimental evidence-based prior knowledge databases [22,23], including miRNA-target mRNA relations and miRNA upstream regulators (e.g., upstream signaling proteins, transcription factor binding sites (TFBSs) in miRNA promoters). The use of cancer contexts
[24] provided further biological interpretability for the network construction. To simplify the network, we determined the underlying substructures (henceforth, network clusters). Notably, in addition to the known miR-221/222-mediated network cluster [9-11], we identified novel miRNA-related network clusters associated with antiestrogen-resistant breast cancer [9-11]. Interestingly, the novel network clusters contained genomic instability, a recently described hallmark of cancer [24] and area of intense interest in the breast cancer field [25].

\section{Results and discussion \\ Overview}

Our goal was to identify a global miRNA-regulated landscape in drug-resistant breast cancer cell lines (MCF7-F, MCF7-T) compared to MCF7 (Additional file 1). To improve the reliability of our approach, experimentally validated miRNA-related databases were used to construct an "evidence-based" miRNA-mRNA network. The network consisted of miRNAs, their targets, transcription factors (TFs) binding to miRNA promoters, and signaling molecules upstream of miRNAs. We further expanded the network by associating it with biological contexts, including the important hallmarks of cancer (henceforth, cancer contexts) [24]. The associations were inspected by utilizing a text-mining tool, PubGene [26]. Subsequently, the expression data for both miRNAs and mRNAs [2] were incorporated into the network. In order to observe differential usage (or identical usage) of the fully integrative network, we examined two different drug resistance states (MCF7-T, MCF7-F) and then refine topologically important network clusters underscoring the network. The details are described in Methods.

\section{Integrative network description}

The integrative network (Figure 1) demonstrated that the entries (e.g., miRNAs, mRNAs) were extensively interconnected with eight cancer-related hallmarks [24], suggesting that miRNA-driven epigenetic changes in these hallmarks [27] contributed to the resistance of MCF7 cells to antiestrogens. Furthermore, both common and differential network usages were observed between the two drug resistance states. To validate the network regulations (Figure 1), we used the mean correlation across all the edges (in the network) as a statistic [19] and performed 5000 node label permutations. As a result, the regulations or the associations in the network were statistically significant at p-value 0.000 (see the details in Additional file 2).

\section{Network cluster analysis}

To further identify important network clusters associated with tamoxifen and fulvestrant resistance, we dissected the integrative network by using a Cytoscape [28] plug-in, 


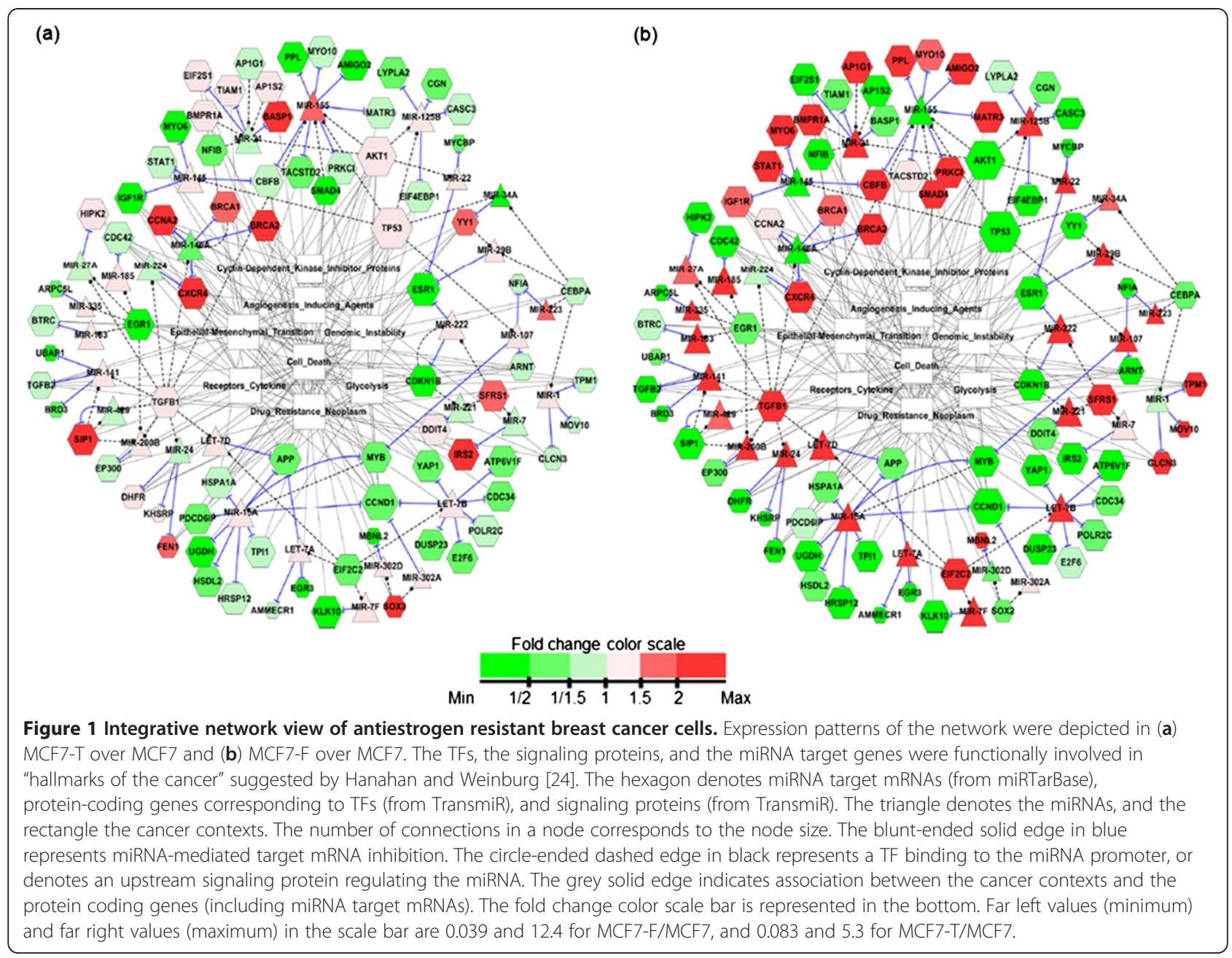

clusterMaker [29]. This analysis resulted in 19 nonorphan network clusters. We then selected the top 10 largest clusters having at least five elements (Figure 2). As cluster 1 showed the strong associations with cancer hallmarks, we examined this cluster in greater detail (below).

Cluster 1 contained both differential and common usages by miRNAs and their targets. The miRNAs and mRNAs relating to the two drug resistant states were associated specifically with various cancer contexts (genomic instability, cell death, epithelial-mesenchymal transition (EMT), drug resistance neoplasm, glycolysis, receptors cytokine) [24]. Mir-146a was down-regulated in both drug resistant cell lines, and its targets, BRCA1/ 2, were up-regulated in the cluster. Mir-146a is considered to function as a tumor suppressor [30,31] in a tissue-dependent context [32], while BRCA1/2 are well known tumor suppressors involved in maintaining genome-integrity, cell cycle, and DNA damage response (DDR) [33,34]. Despite up-regulation of these tumor suppressor genes (TSGs), which would contribute to increased genome stability, it is possible that TSG binding partners could negatively affect genome maintenance [34]. Therefore, we examined expression changes of BRCA1/2 partners in the two resistant cells compared to MCF7. As shown in Additional file 3, expression of BRCA1/2 binding partners was deregulated [33,34]. A recent study [35] proposed that excess of error-free homologous recombination (HR), a critical process for maintaining genomic integrity, can result in genomic instability and that finetuning may be coordinated in the DDR pathway, DNA repair, chromosome segregation, and cell cycle control. Thus, during development of resistance to tamoxifen and fulvestrant, BRCA1/2 binding partner expression changes may be detrimental not only to cell cycle checkpoint [33] but also to the DDR pathway, an important pathway in genomic stability [34]. Although TSGs BRCA1 and BRCA2 were up-regulated in MCF7-F and MCF7-T compared to the parental MCF7 cell line, BRCA1/2 up-regulation may affect fine-tuning of genomic integrity [35].

As mutations are also known to be critical for drug resistance [36,37], we examined publicly known somatic mutations associated with cluster 1 . Somatic mutation of 


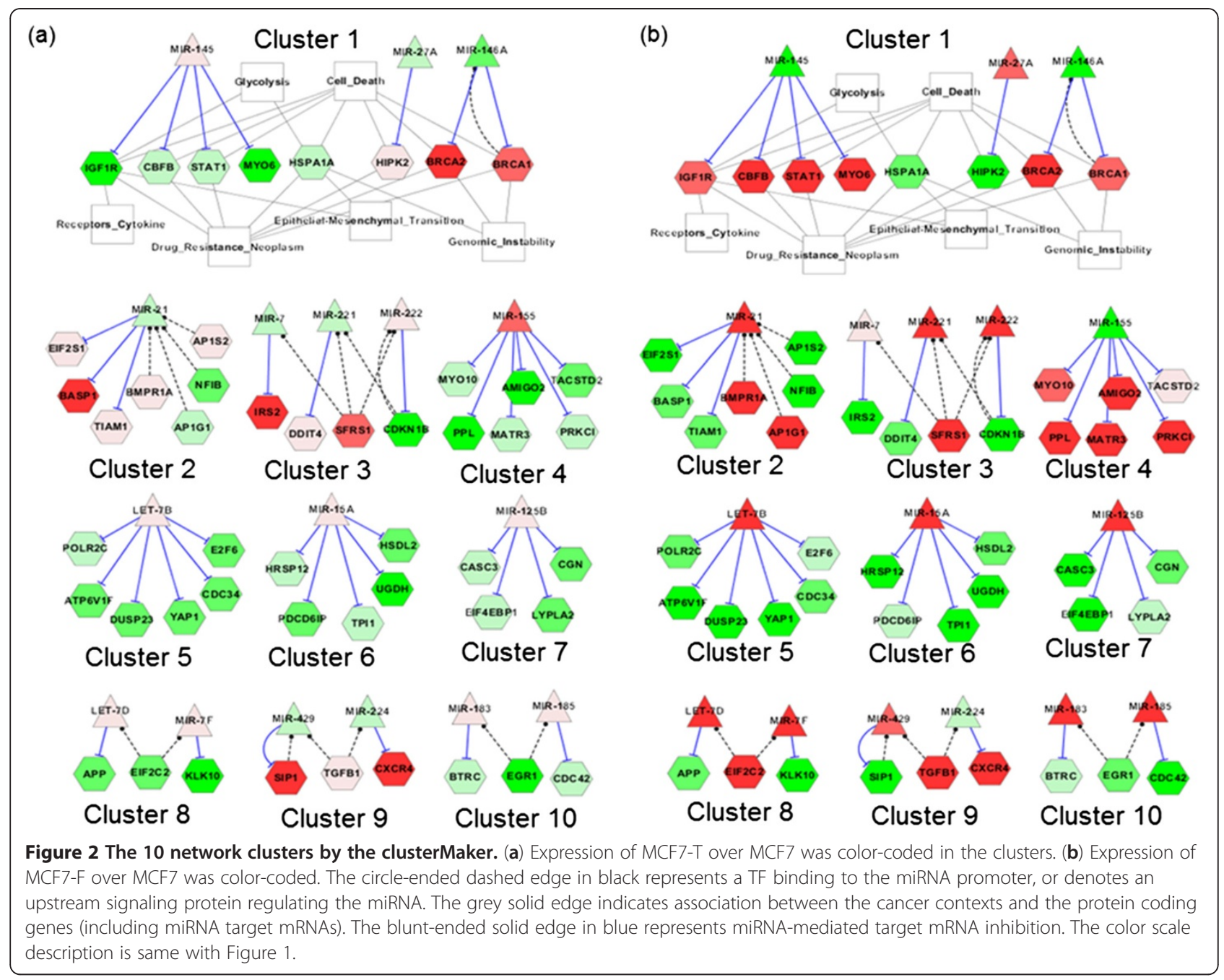

PIK3CA in MCF7 has been reported in the COSMIC database (www.sanger.ac.uk/genetics/CGP/cosmic/) [38] and recent studies [36,37] have reported an association between PIK3CA mutation and breast cancer drug resistance. Our analysis of the two sublines indicates that PI3K/ AKT mutation resulting in signaling activation may contribute to the development of antiestrogen resistance. Such PI3K/AKT activation may also induce phosphorylation, ubiquitination, and nuclear export of CHK1 [39], resulting in genomic instability. We further speculate that PIK3CA mutation in MCF7 cells could acquire gain-offunction (e.g., PI3K/AKT activation) during development of antiestrogen resistance, resulting in genomic instability and contributing to the resistant phenotypes. Interestingly, AKT1 activation has been reported to cause genomic instability via nuclear localization of BRCA1 [40], and PI3KCA (upstream of AKT1) mutation could affect DDR pathway by Brcal protein localization.

MiR-27a and miR-145, by regulating their targets, were involved in MeSH terms EMT, glycolysis, drug resistance neoplasm, cell death, and receptors cytokines. Mir-27a is known to induce paclitaxel-resistance in ovarian cancer cells by targeting HIPK2, followed by reduced MDR1 expression [41]. Thus, it is tempting to speculate that fulvestrant resistance may possibly share the same mechanism with paclitaxel. Mir-145 was slightly up-regulated in MCF7-T and down-regulated in MCF7-F. By repressing various targets, mir-145 has been known to negatively regulate EMT and metastasis $[3,42]$ via STAT1 and IGF1R $[43,44]$. In our previous study [2], we validated that MCF7-F cells had not only reduced cell-cell contacts but increased malignant morphology and characteristics (e.g., increased migration and invasion) compared to the other two cell lines (MCF7, MCF7-T), indicating that MCF7-F underwent EMT and further malignancy. Cluster 1 further indicates a key role for miR-145 down-regulation in fulvestrant resistance, perhaps due to altered regulation of EMT and malignant progression. In addition, because IGF1R is not only an upstream regulator of metabolism (e.g., glycolysis) but 
also regulates STAT1, miR-145 may represent a key regulator of both metabolism and a signaling pathway (JAK/STAT pathway). In summary, cluster 1 shows that miR-146a and BRCA1/2 are shared molecular characteristics of the tamoxifen and the fulvestrant resistances (genomic stability fine-tuning, PI3KCA mutation) and are closely associated in terms of the DDR pathway (e.g. HR). MiR-145 may contribute to the differences in epigenetic background between the two distinct forms of antiestrogen resistance.

Clusters 2 and 4 describe the differential two drugresistant states in terms of miRNA-target interaction, because the two miRNAs (miR-21, miR-155) and their targets show opposite expression. In particular, miR-21 plays an important role both in cancer as well as in stem cell biology [45], inducing EMT and thus contributing to migration, invasion, and morphological change. MiR-21 up-regulation is also consistent in the cell morphology changes associated with acquired antiestrogen resistance [2]. MCF7-T cells grow as tightly packed colonies with limited cell spreading, while MCF7-F cells, by contrast, show reduced cell-cell contacts compared with MCF7 or MCF7-T cells and are loosely attached to the culture surface. Thus, the target genes of the miRNA in this cluster (TIAM1, BASP1, EIF2S1) [46,47] could be involved in EMT as well as in malignant transformation of MCF7-F. In addition, expression of the TFs (AP1S2, AP1G1) upstream of miR-21 was differentially changed between the two drug resistant cell lines and the differential TF usage could also contribute expression changes of this miRNA.

In cluster 4, mir-155 and its targets showed opposite expression patterns in MCF7-T and MCF7-F and thus could contribute to the different drug-resistant states. As the majority of studies indicate that miR-155 is an oncogene [48], this miRNA could play a role in acquired resistance to tamoxifen. However, depending on the biological context, it has been suggested that miR-155 may have a tumor suppressor role $[49,50]$. Thus, we cannot exclude the possibility that different cellular contexts could also contribute to the distinct drug-resistant phenotypes.

Clusters 5, 6, and 7 have the same expression patterns between MCF7-T and -F, implying that the three clusters could be involved in common molecular mechanisms underlying acquired resistance to SERMs and SERDs. Let-7b, miR-15a, and miR-125b were up-regulated in both antiestrogen-resistant cell lines. In particular, miR$125 \mathrm{~b}$ has been reported to play important roles in drugresistance [51] and may be an oncogene [52] in breast cancer. Experimental validation of the functional roles of miR-125b target mRNAs (CGN, CASC3, EIF4EBP1, LYPLA2) during acquired antiestrogen resistance is ongoing in our laboratory.
Clusters 3 and 9 were of interest in terms of mutual regulatory loops. The miR-222 and CDKN1B (p27Kip1) loop in cluster 3 was conserved in MCF7-T and -F in terms of expression, while SIP1 and miR-429 in cluster 9 showed an opposite expression pattern. In particular, miR-222 [9-11] in cluster 3 is closely aligned with ER $\alpha$ status and antiestrogen resistance.

\section{Conclusions}

We applied a systems biology approach [15,53] to MCF7-derived drug resistant cell lines by utilizing miRNAs, mRNAs and text-mining. By using the systems approach to examine global miRNA-target mRNA network in the context of the hallmarks of cancer [24], we identified several important network clusters involved not only in antiestrogen resistance mechanisms but also in differentiating resistance to SERMS and SERDS. To date, only miR-221/222 has been shown to be involved in SERM- and SERD-resistance as well as in ER $\alpha$ status. Our computation reveals that multiple microRNArelated network clusters (Figure 2), in addition to the miR-221/222 network cluster, may contribute to antiestrogen resistant breast cancer cells. Recent reports $[54,55]$ appropriately advocate the need for validating miRNA microarray data. Despite this limitation of the current study, we utilized miRNA-oriented network as well as cancer contexts to implement a systems biology approach in the field of breast cancer and antiestrogen resistance.

\section{Methods \\ Microarray analysis of mRNA and microRNA in MCF7, MCF7-F, and MCF7-T cells}

ER $\alpha$-positive MCF7 cells and their tamoxifen- (MCF7-T) and fulvestrant-resistant (MCF-F) daughter cells were cultured as previously described [2]. For gene expression studies, total RNA was isolated using RNeasy Mini Kits (Qiagen, Valencia, CA, USA), converted to cRNA, labeled and hybridized to Affymetrix U133 Plus 2.0 arrays (Affymetrix, Santa Clara, CA, USA) by the Indiana University Center for Medical Genomics. For microRNA isolation, pelleted cells were resuspended in TRIzol reagent (Invitrogen, Carlsbad, CA, USA) (which retains small RNA molecules), subjected to polyethylene glycol precipitation, RNA ligase-mediated labeling, and hybridized to a custom array, according to previously published method [56]. Quantile normalization [57] was used in both mRNA and miRNA microarrays (Additional files 4 and 5). Each cell line had two replicates in both miRNA and gene expression microarrays.

\section{Databases}

We downloaded the miRNA-related databases $[22,23]$ in order to analyze miRNA-mRNA relations. For better 
accuracy, manually annotated databases, miRTarBase (release 4.2) [22] and TransmiR (v1.1) [23] including only experimentally validated evidences based on literatures, were considered in the study. TransmiR [23] contains upstream regulators (TFs or upstream signaling molecules) controlling miRNA transcription, and miRTarBase [22] has the mRNA targets of miRNAs. The different gene identifier usages in the two databases were converted to gene symbols by using UCSC Genome Browser [58]. The expressions (fold changes) of mRNAs and miRNAs belonging to the two databases were obtained from both miRNA and mRNA microarrays.

\section{Network construction}

We combined the relationships between miRNAs and their upstream regulators (from TransmiR) with those between miRNAs and their target genes (from miRTarBase). In network construction, we restricted the target genes to TF-encoding genes and signaling protein-encoding genes because the two entities, TFs and miRNAs, play important roles in regulation, propagation and cellular fate [59]. We defined the integrated relation, "upstream regulatormiRNA-target", as a circuit. We applied a well-known condition [14], inverse expression between miRNAs and their targets, for further refining the circuits based on the microarray expressions.

Integration of cancer biology-related contexts and circuits PubGene (www.pubgene.org) [26] was used to connect cancer-related context with the regulators (e.g., TFs, signaling molecules) and targets of miRNAs. The contexts were obtained from the hallmarks of cancer proposed by Hanahan and Weinberg [24]. The contexts are related to sustaining proliferative signaling, evading growth suppressors, deregulating cellular energetics, resisting cell death, genome instability, inducing angiogenesis, activating invasion and metastasis. It is noted that immunosuppression, one of the hallmarks, was not inspected because immune cells are not included in the in vitro cell culture conditions. The hallmarks were mapped to appropriate $\mathrm{MeSH}$ (www.ncbi.nlm.nih.gov/mesh) terms in the PubGene: mutation, cyclin dependent kinase inhibitor proteins, cell death, receptors cytokine, drug resistance neoplasm, angiogenesis inducing agents, glycolysis, epithelial-mesenchymal transition, and genomic instability. We added "mRNA-MeSH term" relations (grey solid lines in Figures 1 and 2) into the network of the circuits obtained from the previous section. The integrative network of the circuits and $\mathrm{MeSH}$ terms was constructed and subsequently visualized on Cytoscape [28]. In order to obtain the network clusters, the clusterMaker [29] was applied to the integrative network. Each node was color-coded by its fold-change of its subline (MCF7-F or MCF7-T) over its parental cell line (MCF7).

\section{Additional files}

\begin{abstract}
Additional file 1: Study overview. Integrative network with miRNAs, mRNAs, expressions, and cancer-related contexts in acquired resistance to antiestrogen in breast cancer cells (tamoxifen resistant (MCF7-T),

fulvestrant resistant (MCF7-F), and parental drug-sensitive MCF7 cells). For minimizing false positives in network connectivity, we used

experimentally validated databases: TransmiR (TFs binding in miRNA promoters, signaling proteins for regulating miRNAs), and miRTarBase (miRNA target information). In addition, biological interpretability was enhanced by incorporating cancer-related context terms suggested by Hanahan and Weinberg [24] into the network connectivity. The cancer contexts were connected with TFs, signaling proteins, and miRNA targets from the two databases by using a text-mining tool, PubGene [26]. The antiestrogen resistant cell line expressions were incorporated into the network connectivity (see the details in the Methods section), and the network clusters underscoring the antiestrogen resistances were identified by the Cytoscape clusterMaker [29].
\end{abstract}

Additional file 2: Statistical validation of the regulations in the network. The detailed information is described in this material.

Additional file 3: Genes involved in molecular mechanisms of DNA damage response via BRCA1 and BRCA2. The genes listed refer to Roy et al. [34].

Additional file 4: Fold-change of mRNAs. The fold changes of MCF7-T over MCF7 and MCF7-F over MCF7 were summarized from our previous study [2]. The positive fold change means that the drugresistant cell line is greater than MCF7, and the negative fold change vice versa.

Additional file 5: The processed expression of miRNA microarrays. The expression values were log2-transformed.

\section{Competing interests}

Authors declare no potential competing interests.

\section{Authors' contributions}

SN and KPN conceived and designed the study. SN performed the bioinformatics analysis. CK participated in the Cytoscape analysis. SN, XL, SK and KPN drafted the manuscript. All authors read and approved the final manuscript.

\section{Acknowledgements}

This work is supported by Korean National Cancer Center (NCC) Grant (NCC-1210460 to SN), National Cancer Institute awards (CA113001 and CA085289 to KPN and SK), Natural Science Foundation of China (grant no. 30873044 to $\mathrm{XL}$ ). The microarray studies were carried out using the facilities of the Center for Medical Genomics at Indiana University School of Medicine. The Center for Medical Genomics is supported in part by the Indiana Genomics Initiative of Indiana University, INGEN, which is supported in part by the Lilly Endowment, Inc. Korea Institute of Science and Technology Information kindly provided SN with a computing resource. Drs. Yon Hui Kim and Hae Ryung Chang at NCC helped language editing and critical reading.

\section{Author details}

${ }^{1}$ Cancer Genomics Branch, National Cancer Center, Goyang-si, Gyeonggi-do 410-769, Korea. ${ }^{2}$ Department of Cellular and Integrative Physiology, Medical Sciences Program, Indiana University School of Medicine, Bloomington IN 47405, USA. ${ }^{3}$ Zhongnan Hospital, Wuhan University, Wuhan 430071, China. ${ }^{4}$ Department of Computer Science and Engineering, Bioinformatics Institute, Seoul National University, Seoul 151-742, Korea.

Received: 13 October 2012 Accepted: 20 December 2012

Published: 27 December 2012

\section{References}

1. Osborne CK, Schiff R: Mechanisms of endocrine resistance in breast cancer. Annu Rev Med 2011, 62:233-247.

2. Fan M, Yan PS, Hartman-Frey C, Chen L, Paik H, Oyer SL, Salisbury JD, Cheng AS, Li L, Abbosh PH, et al: Diverse gene expression and DNA methylation profiles correlate with differential adaptation of breast cancer cells to 
the antiestrogens tamoxifen and fulvestrant. Cancer Res 2006, 66:11954-11966.

3. Esquela-Kerscher A, Slack FJ: Oncomirs - microRNAs with a role in cancer Nat Rev Cancer 2006, 6:259-269.

4. Bang-Berthelsen $\mathrm{CH}$, Pedersen L, Floyel T, Hagedorn PH, Gylvin T, Pociot F: Independent component and pathway-based analysis of miRNAregulated gene expression in a model of type 1 diabetes. BMC Genomics 2011, 12:97.

5. Hulf T, Sibbritt T, Wiklund ED, Bert S, Strbenac D, Statham AL, Robinson MD, Clark SJ: Discovery pipeline for epigenetically deregulated miRNAs in cancer: integration of primary miRNA transcription. BMC Genomics 2011, 12:54.

6. Zhou JY, Ma WL, Liang S, Zeng Y, Shi R, Yu HL, Xiao WW, Zheng WL: Analysis of microRNA expression profiles during the cell cycle in synchronized HeLa cells. BMB Rep 2009, 42:593-598.

7. Klinge CM: miRNAs and estrogen action. Trends Endocrinol Metab 2012 23:223-233.

8. Majumder S, Jacob ST: Emerging role of microRNAs in drug-resistant breast cancer. Gene Expr 2011, 15:141-151.

9. Rao X, Di Leva G, Li M, Fang F, Devlin C, Hartman-Frey C, Burow ME, Ivan M, Croce CM, Nephew KP: MicroRNA-221/222 confers breast cancer fulvestrant resistance by regulating multiple signaling pathways. Oncogene 2011, 30:1082-1097.

10. Miller TE, Ghoshal K, Ramaswamy B, Roy S, Datta J, Shapiro CL, Jacob S, Majumder S: MicroRNA-221/222 confers tamoxifen resistance in breast cancer by targeting p27Kip1. J Biol Chem 2008, 283:29897-29903.

11. Di Leva G, Gasparini P, Piovan C, Ngankeu A, Garofalo M, Taccioli C, lorio MV, Li M, Volinia S, Alder H, et al: MicroRNA cluster 221-222 and estrogen receptor alpha interactions in breast cancer. J Natl Cancer Inst 2010, 102:706-721.

12. Nam S, Kim B, Shin S, Lee S: miRGator: an integrated system for functional annotation of microRNAs. Nucleic Acids Res 2008, 36:D159-164

13. Jayaswal V, Lutherborrow M, Ma DD, Yang YH: Identification of microRNAmRNA modules using microarray data. BMC Genomics 2011, 12:138.

14. Nam S, Li M, Choi K, Balch C, Kim S, Nephew KP: MicroRNA and mRNA integrated analysis (MMIA): a web tool for examining biological functions of microRNA expression. Nucleic Acids Res 2009, 37:W356-362.

15. Pe'er D, Hacohen N: Principles and strategies for developing network models in cancer. Cell 2011, 144:864-873.

16. Dutkowski J, Ideker T: Protein networks as logic functions in development and cancer. PLOS Comput Biol 2011, 7:e1002180.

17. Barabasi AL, Oltvai ZN: Network biology: understanding the cell's functional organization. Nat Rev Genet 2004, 5:101-113.

18. Baliga NS, Pan M, Plaisier CL: A miRNA-regulatory network explains how dysregulated miRNAs perturb oncogenic processes across diverse cancers. Genome Res 2012, 22:2302-2314.

19. Nunez-lglesias J, Liu CC, Morgan TE, Finch CE, Zhou XJ: Joint genome-wide profiling of miRNA and mRNA expression in Alzheimer's disease cortex reveals altered miRNA regulation. PLoS One 2010, 5:e8898.

20. Ferraro L, Ravo M, Nassa G, Tarallo R, De Filippo MR, Giurato G, Cirillo F, Stellato C, Silvestro S, Cantarella C, et al: Effects of oestrogen on microRNA expression in hormone-responsive breast cancer cells. Horm Cancer 2012, 3:65-78.

21. Manavalan TT, Teng Y, Appana SN, Datta S, Kalbfleisch TS, Li Y, Klinge CM: Differential expression of microRNA expression in tamoxifen-sensitive MCF-7 versus tamoxifen-resistant LY2 human breast cancer cells. Cancer Lett 2011, 313:26-43.

22. Hsu SD, Lin FM, Wu WY, Liang C, Huang WC, Chan WL, Tsai WT, Chen GZ, Lee CJ, Chiu CM, et al: miRTarBase: a database curates experimentally validated microRNA-target interactions. Nucleic Acids Res 2011, 39:D163-169.

23. Wang J, Lu M, Qiu C, Cui Q: TransmiR: a transcription factor-microRNA regulation database. Nucleic Acids Res 2010, 38:D119-122.

24. Hanahan D, Weinberg RA: Hallmarks of cancer: the next generation. Cell 2011, 144:646-674

25. Martin SA, Hewish M, Lord CJ, Ashworth A: Genomic instability and the selection of treatments for cancer. J Pathol 2010, 220:281-289.

26. Jenssen TK, Laegreid A, Komorowski J, Hovig E: A literature network of human genes for high-throughput analysis of gene expression. Nat Genet 2001, 28:21-28.
27. Balch C, Nephew KP: The role of chromatin, microRNAs, and tumor stem cells in ovarian cancer. Cancer Biomark 2010, 8:203-221.

28. Smoot ME, Ono K, Ruscheinski J, Wang PL, Ideker T: Cytoscape 2.8: new features for data integration and network visualization. Bioinformatics 2011, 27:431-432.

29. Morris JH, Apeltsin L, Newman AM, Baumbach J, Wittkop T, Su G, Bader GD, Ferrin TE: clusterMaker: a multi-algorithm clustering plugin for Cytoscape. BMC Bioinformatics 2011, 12:436.

30. Lin SL, Chiang A, Chang D, Ying SY: Loss of mir-146a function in hormone-refractory prostate cancer. RNA 2008, 14:417-424.

31. Li Y, Vandenboom TG, Wang Z 2nd, Kong D, Ali S, Philip PA, Sarkar FH: miR-146a suppresses invasion of pancreatic cancer cells. Cancer Res 2010, 70:1486-1495

32. Rusca N, Monticelli S: MiR-146a in Immunity and Disease. Mol Biol Int 2011 , 2011:437301.

33. Deng CX: BRCA1: cell cycle checkpoint, genetic instability, DNA damage response and cancer evolution. Nucleic Acids Res 2006, 34:1416-1426.

34. Roy R, Chun J, Powell SN: BRCA1 and BRCA2: different roles in a common pathway of genome protection. Nat Rev Cancer 2012, 12:68-78.

35. Guirouilh-Barbat JK, Wilhelm T, Lopez BS: AKT1/BRCA1 in the control of homologous recombination and genetic stability: the missing link between hereditary and sporadic breast cancers. Oncotarget 2010 1:691-699.

36. Wang L, Zhang Q, Zhang J, Sun S, Guo H, Jia Z, Wang B, Shao Z, Wang Z, Hu X: PI3K pathway activation results in low efficacy of both trastuzumab and lapatinib. BMC Cancer 2011, 11:11-248.

37. Miller TW, Rexer BN, Garrett JT, Arteaga CL: Mutations in the phosphatidylinositol 3-kinase pathway: role in tumor progression and therapeutic implications in breast cancer. Breast Cancer Res 2011, 13:224.

38. Forbes SA, Bindal N, Bamford S, Cole C, Kok CY, Beare D, Jia M, Shepherd R, Leung $\mathrm{K}$, Menzies $\mathrm{A}$, et al: COSMIC: mining complete cancer genomes in the Catalogue of Somatic Mutations in Cancer. Nucleic Acids Res 2011, 39:D945-950.

39. Puc J, Keniry M, Li HS, Pandita TK, Choudhury AD, Memeo L, Mansukhani M, Murty W, Gaciong Z, Meek SE, et al: Lack of PTEN sequesters CHK1 and initiates genetic instability. Cancer Cell 2005, 7:193-204.

40. Plo I, Laulier C, Gauthier L, Lebrun F, Calvo F, Lopez BS: AKT1 inhibits homologous recombination by inducing cytoplasmic retention of BRCA1 and RAD51. Cancer Res 2008, 68:9404-9412.

41. Li Z, Hu S, Wang J, Cai J, Xiao L, Yu L, Wang Z: MiR-27a modulates MDR1/ P-glycoprotein expression by targeting HIPK2 in human ovarian cancer cells. Gynecol Oncol 2010, 119:125-130.

42. Peng X, Guo W, Liu T, Wang X, Tu X, Xiong D, Chen S, Lai Y, Du H, Chen G, et al: Identification of miRs-143 and -145 that is associated with bone metastasis of prostate cancer and involved in the regulation of EMT. PLoS One 2011, 6:e20341

43. Gregersen $L H$, Jacobsen $A B$, Frankel LB, Wen J, Krogh $A$, Lund $A H$ : MicroRNA-145 targets YES and STAT1 in colon cancer cells. PLOS One 2010, 5:e8836.

44. La Rocca G, Badin M, Shi B, Xu SQ, Deangelis T, Sepp-Lorenzinoi L, Baserga R: Mechanism of growth inhibition by MicroRNA 145: the role of the IGF-I receptor signaling pathway. J Cell Physiol 2009, 220:485-491.

45. Han M, Liu M, Wang Y, Mo Z, Bi X, Liu Z, Fan Y, Chen X, Wu C: Reexpression of miR-21 contributes to migration and invasion by inducing epithelial-mesenchymal transition consistent with cancer stem cell characteristics in MCF-7 cells. Mol Cell Biochem 2012, 363:427-436.

46. Cottonham CL, Kaneko S, Xu L: miR-21 and miR-31 converge on TIAM1 to regulate migration and invasion of colon carcinoma cells. J Biol Chem 2010, 285:35293-35302.

47. Yang Y, Chaerkady R, Beer MA, Mendell JT, Pandey A: Identification of miR-21 targets in breast cancer cells using a quantitative proteomic approach. Proteomics 2009, 9:1374-1384.

48. Jiang S, Zhang HW, Lu MH, He XH, Li Y, Gu H, Liu MF, Wang ED: MicroRNA-155 functions as an OncomiR in breast cancer by targeting the suppressor of cytokine signaling 1 gene. Cancer Res 2010, 70:3119-3127.

49. Li CL, Nie H, Wang M, Su LP, Li JF, Yu YY, Yan M, Qu QL, Zhu ZG, Liu BY: microRNA-155 is downregulated in gastric cancer cells and involved in cell metastasis. Oncol Rep 2012, 27:1960-1966.

50. Dorsett Y, McBride KM, Jankovic M, Gazumyan A, Thai TH, Robbiani DF, Di Virgilio M, Reina San-Martin B, Heidkamp G, Schwickert TA, et al: MicroRNA-155 
suppresses activation-induced cytidine 0deaminase-mediated Myc-lgh translocation. Immunity 2008, 28:630-638.

51. Zhou M, Liu Z, Zhao Y, Ding Y, Liu H, Xi Y, Xiong W, Li G, Lu J, Fodstad O, et al: MicroRNA-125b confers the resistance of breast cancer cells to paclitaxel through suppression of pro-apoptotic Bcl-2 antagonist killer 1 (Bak1) expression. J Biol Chem 2010, 285:21496-21507.

52. Budhu A, Ji J, Wang XW: The clinical potential of microRNAs. J Hematol Oncol 2010, 3:37.

53. Pradhan MP, Prasad NK, Palakal MJ: A systems biology approach to the global analysis of transcription factors in colorectal cancer. BMC Cancer 2012, 12:331.

54. Git A, Dvinge H, Salmon-Divon M, Osborne M, Kutter C, Hadfield J, Bertone $P$, Caldas $C$ : Systematic comparison of microarray profiling, real-time PCR, and next-generation sequencing technologies for measuring differential microRNA expression. RNA 2010, 16:991-1006

55. Chen Y, Gelfond JA, McManus LM, Shireman PK: Reproducibility of quantitative RT-PCR array in miRNA expression profiling and comparison with microarray analysis. BMC Genomics 2009, 10:407.

56. Thomson JM, Parker J, Perou CM, Hammond SM: A custom microarray platform for analysis of microRNA gene expression. Nat Methods 2004, 1:47-53.

57. Bolstad BM, Irizarry RA, Astrand M, Speed TP: A comparison of normalization methods for high density oligonucleotide array data based on variance and bias. Bioinformatics 2003, 19:185-193.

58. Zweig AS, Karolchik D, Kuhn RM, Haussler D, Kent WJ: UCSC genome browser tutorial. Genomics 2008, 92:75-84.

59. Hobert O: Common logic of transcription factor and microRNA action. Trends Biochem Sci 2004, 29:462-468.

doi:10.1186/1471-2164-13-732

Cite this article as: Nam et al:: An integrative analysis of cellular contexts, miRNAs and mRNAs reveals network clusters associated with antiestrogen-resistant breast cancer cells. BMC Genomics 2012 13:732.

\section{Submit your next manuscript to BioMed Central and take full advantage of:}

- Convenient online submission

- Thorough peer review

- No space constraints or color figure charges

- Immediate publication on acceptance

- Inclusion in PubMed, CAS, Scopus and Google Scholar

- Research which is freely available for redistribution 